\title{
Diffuse lymphocytic lymphoma with splenic rupture
}

\author{
W. H. F. THOMSON \\ M.B., F.R.C.S.(Eng.) \\ Surgical Registrar, \\ Redhill General Hospital
}

WHEN the splenic capsule is found to be lacerated or the spleen avulsed from its pedicle, it is almost always due to recent injury. Occasionally, a patient will present as an acute abdominal emergency with or without the signs of haemorrhage but no history of recent injury, and at laparotomy, rupture of the spleen will be found. If, in such circumstances, careful questioning has failed to reveal a history of trauma or unusual effort, and on examination of the spleen, both macroscopically and microscopically, no abnormality apart from the haemorrhage and laceration can be found, then such an example can be truly labelled spontaneous rupture of the normal spleen. Such an event is but rarely reported. Orloff \& Peskin in 1958, after reviewing seventy-one reported cases in the literature, considered only twenty to be fully acceptable. Seven years later, Naiberg and his colleagues (Naiberg, Sidlofsky \& Chris, 1965) reported a case conforming to the above criteria but were able to add only two further examples.

Much commoner is sudden rupture of the diseased spleen and this event has been recorded in a wide range of splenic diseases. In this context, a controversial point which recurs in the literature concerns the interpretation of the meaning of the term 'spontaneous'. Whereas in 1964, Hynes, Silverstein \& Fawcett found seventeen examples of spontaneous rupture of the spleen in leukaemia, Stiles \& Ultman in 1966 in reviewing thirty-two cases of rupture of the leukaemic spleen accepted only eight as being truly spontaneous in origin.

The following is a case report of rupture of the diseased spleen in the absence of any trauma.

\section{Case report}

A publican aged 45 was admitted to hospital with a 5-month history of pains in the legs, abdomen and back and more recently, spontaneous bruising, rectal bleeding and haematuria. There was no history of exposure to any drugs or chemicals and no family history of purpura.

Examination revealed purpura and a spleen palpable to 4 finger breadths below the left costal margin. No lymphadenopathy or hepatomegaly was found.
Investigations: $\mathrm{Hb} 101 \%(14.8 \mathrm{~g} / 100 \mathrm{ml})$, WBCs $5400 / \mathrm{mm}^{3}$ (neutrophils $14 \%$, lymphocytes $85 \%$, eosinophils $1 \%$ ) and platelets $12,000 / \mathrm{mm}^{3}$. Examination of the marrow aspirate showed a moderately cellular picture with normal erythrocyte production. A reduction in the granulocyte series with an occasional large myelocyte was seen. Small smear cells were also present. Megakaryocytes were present in normal numbers. These investigations indicated that the thrombocytopaenia was caused peripherally. Prednisolone $60 \mathrm{mg}$ daily, at first efficacious, increased the platelet count, but 19 days after commencing treatment this count had fallen to $4200 / \mathrm{mm}^{3}$. Splenectomy was then advised because of the failure of prednisolone to control the thrombocytopaenia, presumed due to hypersplenism, and because histological examination of the spleen might well give the diagnosis of the underlying disease Two days prior to elective splenectomy, while lying. in bed, he suddenly developed abdominal distention and pain in the left shoulder. A diagnosis of retroperitoneal haemorrhage was made and laparotomy advised.

At operation the peritoneal cavity was found to contain $500 \mathrm{ml}$ of fresh blood and there was a tear on the postero-medial border of the enlarged spleen. Splenectomy was performed and he made an uninterrupted recovery, the platelet count rising steadily to normal levels. Histological examination of the spleen showed complete destruction of the architecture by infiltration with primitive lymphocytes. This picture, in association with an increase in lymphocytes present in the marrow and peripheral blood, was that of diffuse lymphocytic lymphoma or the aleukaemic form of chronic lymphocytic leukaemia.

After splenectomy, the patient was carefully questioned about the possibility of trauma but could recall none in either the immediate or remote past.

\section{Discussion}

Considering the ease with which the capsule of the normal spleen can be torn during operative procedures in the upper left quadrant of the abdomen, particularly tears in the region of the lieno-colic ligament when the spleen is moved in relation to the 
splenic flexure or vice versa, how much more susceptible to injury the spleen will be when its architecture has been disrupted and weakened by a disease. Thus an everyday occurrence, such as coughing, bending or sneezing would be likely to initiate a tear in the splenic capsule. Such a physiological event may not be remembered by the patient, let alone recorded by the clinician so that it would seem to be preferable when the spleen is diseased, to avoid the use of the term 'spontaneous'. This is not to deny that truly spontaneous rupture of the diseased spleen occurs, but to stress the difficulty in differentiating between this and rupture of a diseased, parlously intact spleen, precipitated by minor stress which would have no effect on a normal spleen. Walton (1963) suggests the description 'pathological rupture' in these circumstances.

The aetiology of splenic rupture in leukaemia and the reticuloses in the absence of trauma may depend on one or more factors. Firstly, defects in blood coagulability must play a part. The patient reported had already bled spontaneously into the kidneys, skin and intestine and so it can be appreciated how a haematoma could have developed and subsequently ruptured through the splenic capsule. Secondly, if the splenic capsule is distended and infiltrated by a leukaemic or lymphomatous process then rupture is likely, particularly if there are adhesions to the bowel and diaphragm, which may be stretched by peristalsis and respiration. Lastly, infarction of the spleen may occur in leukaemia and the reticuloses with consequent rupture.

\section{Acknowledgments}

This patient was under the care of Dr Paget Davies ard Mr A. E. Stevens and I am grateful to them for their helpful criticism in the preparation of this paper.

\section{References}

Hynes, H.E., Silverstein, M.N. \& Fawcett, K.J. (1964) Spontaneous rupture of the spleen in acute leukaemia: report of 2 cases. Cancer, 17, 1356.

NaIberg, D., Sidlofsky, S. \& Chris, S.M. (1965) Spontaneous rupture of the spleen. Can. J. Surg. 8, 96.

Orloff, M.J. \& Peskin, G.W. (1958) Spontaneous rupture of the normal spleen-a surgical enigma. Int. Abstr. Surg. 106, 1 .

Stiles, T.B. \& UltmanN, J.E. (1966) Spontaneous rupture of the spleen in chronic lymphocytic leukaemia. Cancer, $19,1587$.

Walton, T.T. (1963) Splenic rupture in infectious mononucleosis. Texas J. Med. 59, 333.

\title{
Spontaneous rupture of the spleen in the prodromal period of chickenpox
}

\author{
HaziQ ul YaQIN \\ B.Sc., M.B., B.S., F.R.C.S., F.R.C.S.E. \\ Surgical Registrar, \\ Stoke Mandeville Hospital, Aylesbury
}

SPONTANEOUS rupture of the spleen is a rare surgical emergency but is a known hazard of pathological splenomegaly. It can occur in patients with an apparently normal spleen (Susman, 1927; Coleman, 1939; Orloff \& Peskin, 1958; Brodman \& Bautista, 1959).

Since Atkinson's report in 1874 a large number of cases of spontaneous rupture of the spleen have been described. It has occurred in malaria, kala-azar, infectious mononucleosis, typhoid, leukaemia and typhus; and less commonly in portal thrombosis, splenic infarct, Hodgkin's disease, splenic abscess, Banti's disease, relapsing fever, puerperal sepsis, pneumonia and certain other acute infective conditions.

This report describes spontaneous rupture of the spleen occurring in a young adult male who was suffering from prodromal chickenpox. A thorough search of the literature has failed to reveal any record of a similar case.

\section{Case report}

The patient, a 25-year-old warehouseman, was admitted to hospital as an emergency on 27 March 1968. Previous to this episode of illness, he was in good health and at full work.

On admission he complained of severe lower abdominal pain associated with nausea of $10 \mathrm{hr}$ duration. A day prior to his admission to the hospital he felt feverish and had backache and did not go to work. At 03.00 hours on the day of admission he was awakened by very severe generalized abdominal pain. This pain was accompanied by severe nausea and retching, but the patient did not 